\title{
A Comparison between Effects of Linear and Non-linear mechanistic behaviour of materials on the Layered Flexible Pavement Response
}

\author{
Behzad Ghadimi ${ }^{1, a}$, Hamid Nikraz ${ }^{2, b}$, Colin Leek ${ }^{3, c}$ and Ainalem Nega ${ }^{4, d}$
}

\begin{abstract}
${ }^{1}$ PhD Candidate, Department of Civil Engineering, Curtin University, GPO Box U1987, Perth, WA 6845, Australia; Tel: (+61) 8 9266-2674; Fax: (+61) 8 9266-2681; Email: Behzad.Ghadimi@ postgrad.curtin.edu.au

${ }^{2}$ Professor, Head of Department of Civil Engineering, Curtin University, GPO Box U1987, Perth, WA 6845, Australia; Tel: (+61) 8 9266-7573; Fax: (+61) 8 9266-2681; Email: H.Nikraz@ @urtin.edu.au

${ }^{3}$ Adjunct Professor, Department of Civil Engineering, Curtin University, GPO Box U1987, Perth, WA 6845, Australia; Tel: (+61) 8 9266-4761; Fax: (+61) 8 9266-2681; Email: C.Leek@ curtin.edu.au

${ }^{4}$ PhD Candidate, Department of Civil Engineering, Curtin University, GPO Box U1987, Perth, WA 6845, Australia; Tel: (+61) 8 9266-2674; Fax: (+61) 8 9266-2681; Email: Ainalem.Nega@curtin.edu.au
\end{abstract}

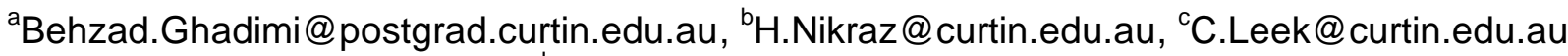 \\ dAinalem.Nega@curtin.edu.au
}

Keywords: Numerical modeling, ABAQUS, CIRCLY, KENLAYER, Flexible Pavement.

\begin{abstract}
Modelling granular pavement materials has a significant role in the pavement design procedure. Modelling can be through an experimental or numerical approach to predict the granular behaviour during cyclic loading. The current design process in Australia is based on linear elastic analysis of layers. The analysis is performed through a well-known program CIRCLY which is applied to model bound pavement material behaviour. The KENLAYER is one of the common pavement software models used for pavement design in the United States which performs non-linear analysis for granular materials. Alternatively, a general Finite Element program such as ABAQUS can be used to model the complicated behaviour of multilayer granular materials. This study is to compare results of numerical modelling with these three programs on two sample pavement models.
\end{abstract}

\section{Introduction}

Design and construction procedures for transportation infrastructure such as road pavements, railway formations, and airfield pavements are aimed at assessing the permanent deformations and or fatigue cracking of the bound or unbound layers. Currently there is a growing trend to use computer software in design and analysis of pavement materials; however, each of these programs has its own specific ability and limitations. These computer programs are manipulated to model (called numerical modeling) the behavior of flexible pavements under certain load conditions. Two different approaches are usual: first approach is using an analytical solution provided from theory of elasticity with calibration factors to match empirically observed behavior while the second approach is implementation of finite element techniques to solve the general equilibrium of the whole layered system.

The advantages of numerical models for designers and researches are that they are considerably cheaper and they provide very rapid computation on standard computers. Moreover, in numerical modeling the full layered pavement system behavior can be observed and investigated. 


\section{Review of Linear Elastic Computer Programs: CIRCLY 5.0 and KENLAYER}

Based on linear elastic theory, an analytical solution of a layered semi-infinite half-space can be calculated. The assumptions are that stress-strain behavior is linear elastic and the pavement domain has no limit in horizontal direction. In vertical direction there is a horizontal stress-free surface at top of the medium while the bottom is extended to infinite depth.

Two well-accepted pavement design programs, KENLAYER and CIRCLY, calculate pavement system responses (stress, strain, deformation, etc.) based on elastic theory.

The KENLAYER Computer Program ([1] , [2]) is an accepted computer program which can model pavement layers as linear elastic, nonlinear elastic or as Mohr-Coulomb elastoplastic materials. The main core of KENLAYER is the solution for an elastic multilayer system under a circular loaded area.

The KENLAYER Computer Program can only be used to analyze flexible pavements with no joints. This program can use the superposition principle for multiple wheels. It can also use an iterative technique to solve non-linear problems ([3]) .

D.S Gedafa [3] used KENLAYER and HDM-4 to analyze flexible pavement performance. In this study these two computer programs have been compared. It is concluded that KENLAYER is can be more easily applied to performance analysis while HDM-4 is a more powerful tool in the field of strategic analysis.

CIRCLY ([4]) is a computer program for pavement design and can be applied to material analysis. It can model materials either isotropic or anisotropic. The load is considered as the tyre pressure uniformly distributed over a circular area. The analysis is assumed to be under static condition and the superposition principle is valid.

CIRCLY was developed as a geomechanical program in the Division of Applied Geomechanics of Commonwealth Scientific and Industrial Research Organization (CSIRO) of Australia. Then in 1987, the National Association of Australian State Road Authorities (NAASRA) used CRICLY in the Guide to Structural Design of Pavements. This is the basis for "Guide to Structural Design of Pavements" developed and modified by AUSTROADS (formerly NAASRA) in 1992 and 2004. The CIRCLY 5.0 (current version of the program) is written in FORTRAN IV ([5]).

One of the referred studies in which an interesting comparison has been presented was undertaken by Ullidtz [6]. In this paper six pavement programs including CIRCLY and KENLAYER were compared against measured field data. Field data was collected from three full scale pavement projects from CEDEX in Spain, DTU in Denmark and LAVOC in Switzerland.

A review of the mechanistic design approach in pavement design was undertaken by Wardle et al. [7]. According to Wardle "Unbound layers should be "sub-layered" in order to better model their non-linear response". In this study CIRCLY is manipulated to calculate the vertical strain for 4 and 20 ton wheel loads and the result plotted at varying depths in the pavement.

Hadi and Symons [8] have compared results of the CIRCLY program with a finite element model constructed in MSC/NASTRAN and STRAND. According to their study, CIRCLY resulted in a lower number of allowable repetitions based on Austroads recommended loading.

Tutumluer [9] compared the results of pavement modeling through finite element program GT-PAVE and CIRCLY for a cross anisotropic model. In this study, the two programs were used to calculate the elastic response of layered pavement system. Although both of these programs are based on an analytical solution, they are unable to produce "the exact" results. The reason is that in both programs, the calculation approach is numerical and there is a level of approximation in each.

\section{Review of Abaqus Application in Flexible Pavement Modeling}

Advancing technology in computers is making it more attractive for engineers to use advanced computational methods instead of analytical solutions previously limited by computer power. One of the most accepted methods among the available options is the finite element method 
(FEM). The main advantage of FEM-based programs is their ability to model various types of mechanical loadings and behaviors in a two or three dimensional medium.

The ABAQUS program, a general purpose FEM program, has been used to model layered flexible pavement systems.

Zaghloul and White [10] used ABAQUS to model the three dimensional behavior of a pavement layered system under dynamic loading. Mallela and George [11], Uddin and Pan [12] and Cho et al. [13] also employed ABAQUS in three dimensional pavement models. Kim and Tutumluer ([14], [15] and [16]) have used ABAQUS in their study on modeling the nonlinear behavior of the stress-dependent pavement foundation (subgrade).

Vuong [17] has investigated the effect of repeated loading on pavement granular materials. In this study a nonlinear finite element has been used to predict the stress-strain response of the pavement system. Many loading conditions, including single, tandem, tri and quad axle have been chosen to validate the finite element analysis results.

Bodhinayake [5] has used the ABAQUS to model nonlinearity in subgrade soil while other pavement layers has been modeled as a linear elastic material.

In the current study the ABAQUS software package is used to determine its capacity and compare the output with the KENLAYER and CIRCLY programs.

\section{Numerical Modeling}

While there is a traditional inclination towards laboratory and field tests in pavement engineering, recently the numerical modeling option has attracted many researchers. Duncan et al. [18] first used the finite element approach in flexible pavements analysis. Huang [19] calculated stresses and displacements in nonlinear soil through finite element modeling. Since then many authors have used numerical modeling to calculate induced damage in pavement layers, including the asphalt layer, base and subgrade.

This research is following two main purposes. First is to compare three different programs which use different approaches to predict the behavior of pavement materials. To do so three well-known programs which are: KENLAYER, CIRCLY and ABAQUS are manipulated to construct first sample layered pavement system. A fixed geometry and load condition is chosen for analysis. This step can show the validation of linear finite element model.

Second purpose is to introduce a new method to calculate the nonlinear behavior of granular materials (mainly base and subgrade) used in flexible layered system. This has been done through constructing the second model in ABAQUS. Both linear and nonlinear analyses are executed on this second model and the results are presented and compared.

\section{Linear Model: Methodology and Characteristics}

A sample section of a layered pavement with same thickness, geometry and loading characteristics is modeled in the aforementioned programs. Figure 1 illustrates the geometry of the modeled pavement.

The material properties of the first model are listed in Table 1. All layers are assumed to behave linear elastically under a $0.75 \mathrm{MPa}$ pressure loading, which is applied over a circular area of $92 \mathrm{~mm}$ radius. This is taken as a circular representation of the tyre pressure in the AUSTROADS method employed in CIRCLY ([20]). 


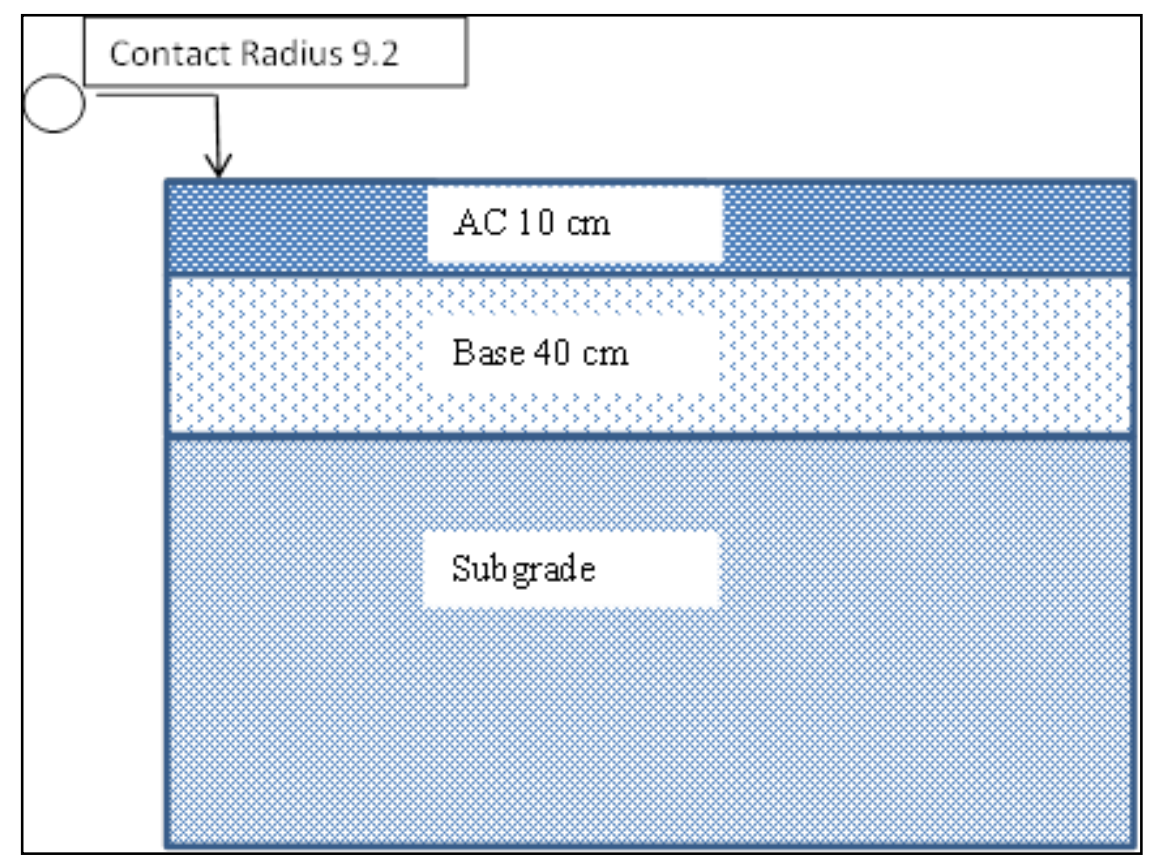

Figure 1. Constructed model in KENLAYER and CIRCLY

Table 1. Material Properties Linear Model

\begin{tabular}{cccc}
\hline$\underline{\text { Layer }}$ & $\frac{\text { Thickness }}{(\mathrm{mm})}$ & $\frac{\underline{\text { Elastic }}}{\text { Modulus }}$ & $\underline{\text { Poisson }}$ \\
\hline$\underline{\text { Asphalt (AC) }}$ & $\underline{100}$ & $\underline{2800}$ & $\underline{\text { Ratio }}$ \\
$\begin{array}{c}\text { Granular } \\
\frac{\text { Base/Subbase })}{\text { Subgrade }}\end{array}$ & $\underline{400}$ & $\underline{500}$ & $\underline{0.35}$ \\
\hline
\end{tabular}

Based on elastic theory under isotropic conditions, in three-dimensional elastic analysis the stresses and strains are related to each other. Equation 1 shows this relation ([21]):

$$
\begin{aligned}
& \varepsilon_{x x}=\frac{1}{E}\left[\sigma_{x x}-v\left(\sigma_{y y}+\sigma_{z z}\right)\right] . \\
& \varepsilon_{y y}=\frac{1}{E}\left[\sigma_{y y}-v\left(\sigma_{x x}+\sigma_{z z}\right)\right] . \\
& \varepsilon_{z z}=\frac{1}{E}\left[\sigma_{z z}-v\left(\sigma_{x x}+\sigma_{y y}\right)\right] .
\end{aligned}
$$

where:

$\sigma$ is the normal stress along the axes.

$\varepsilon$ is the normal strain along the axes.

$\mathrm{E}$ is the elastic modulus of the materials.

$v$ is the Poisson ratio.

However, in this study the models are constructed and analyzed in two dimensional axisymmetric conditions. When axisymmetric conditions are applied, the full three-dimensional equation can be reduced to Equation 2: 


$$
\begin{aligned}
& \varepsilon_{\mathrm{r}}=\frac{1}{\mathrm{E}}\left(\sigma_{\mathrm{r}}-v \sigma_{\mathrm{z}}\right) . \\
& \varepsilon_{\mathrm{z}}=\frac{1}{\mathrm{E}}\left(\sigma_{\mathrm{z}}-v \sigma_{\mathrm{r}}\right) .
\end{aligned}
$$

where:

$\sigma_{\mathrm{r}}$ is the radial stress.

$\sigma_{z}$ is the vertical stress.

$\varepsilon_{\mathrm{r}}$ is the radial strain.

$\varepsilon_{\mathrm{z}}$ is the vertical strain.

$\mathrm{E}$ is the elastic modulus of the materials.

$v$ is the Poisson ratio.

For layered pavement systems, multi-layer elastic theory is applied where the pavement system is considered as horizontally infinite layers. These layers are of determined thickness in the vertical direction. In pavement analysis it is also usual to consider the last layer (subgrade) as a vertically infinite layer. Therefore, the whole system is modeled as multi-layered semi- infinite half space. The other assumption applied in the elastic model used in CIRCLY and KENLAYER is a full friction condition between two consecutive layers. Finally, the surface of pavement system is considered as a frictionless layer causing no shear stress ([5]).

While CIRCLY and KENLAYER use an analytical equation for multi-layered half space medium to simulate the actual condition of pavement structure, the finite element approach tries to predict the mechanical response of the system through solving by equilibrium of forces. The assumption here is that the model should satisfy continuity of medium (no crack is modeled).

Although this method of modeling is more difficult, it has the advantage to model various types of loading conditions, geometry and material behavior. This capacity is implemented in ABAQUS program very effectively.

Figure 2 illustrates the geometry of FEM model. The material properties are the same as listed in Table 1. However the geometry of the model in ABAQUS cannot be the same as CIRCLY and KENLAYER because the horizontal and vertical dimension must be finite. To overcome this problem Duncan et al. [18] suggested a dimension of 50-times $\mathrm{R}$ (loading radius) in vertical and 12-times R in horizontal direction. Kim and Tutumluer [16] found a good agreement between results of the FE analysis and KENLAYER when the model dimension is 140-times $\mathrm{R}$ in vertical and 20-times $\mathrm{R}$ in horizontal direction. In this study, after several trial runs, the final dimension of model has been selected as 55 times $\mathrm{R}$ in horizontal direction and 109 times $\mathrm{R}$ in vertical direction.

Figure 3 shows the constructed FE meshes for the axisymmetric 2D analysis. Vertical lines on both sides of the finite element model are bounded with roller boundary condition which permits the displacement in the vertical direction but prohibits it in the horizontal direction. The base of the model is fixed in every direction. The model contains 3402 biquadratic axisymmetric quadrilateral reduced integration elements and 10453 nodes. 


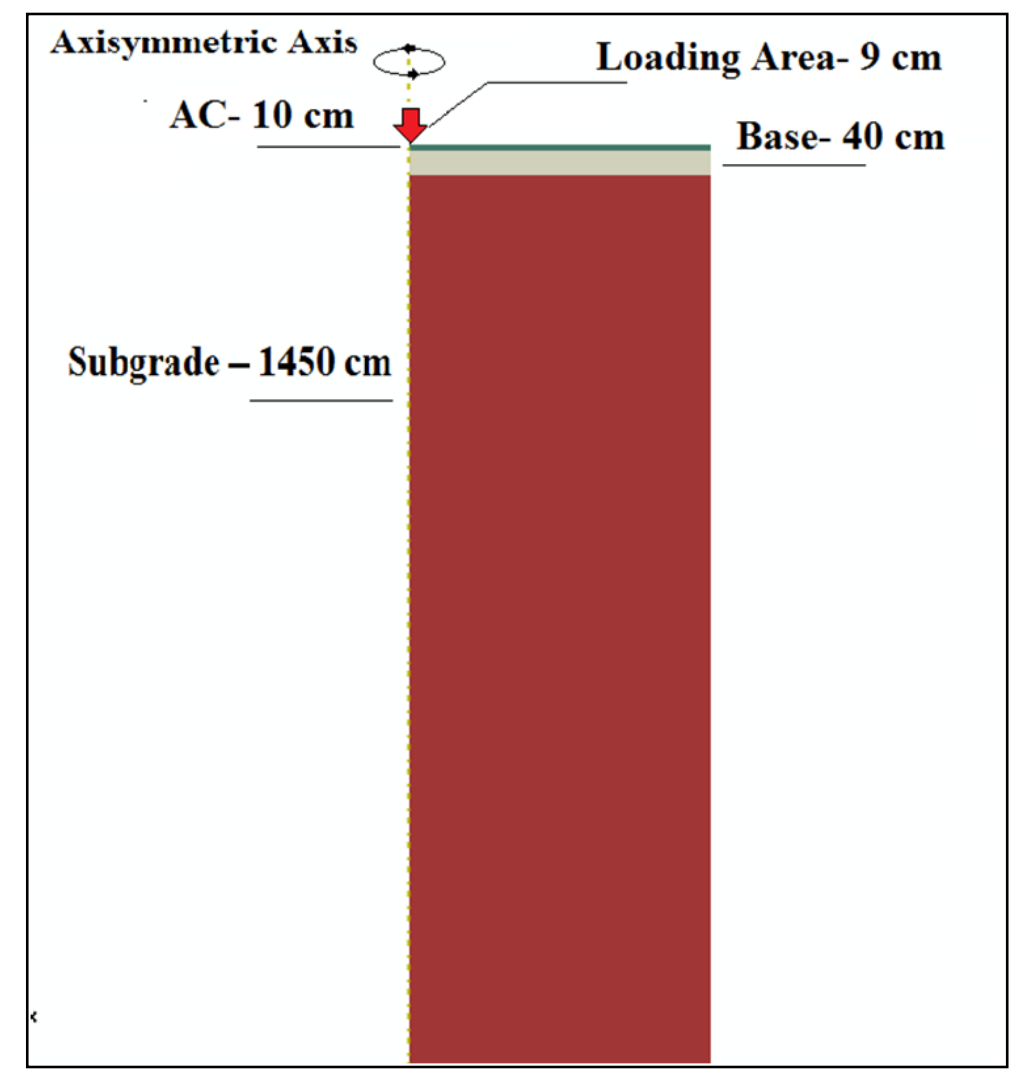

Figure 2. Constructed model in ABAQUS

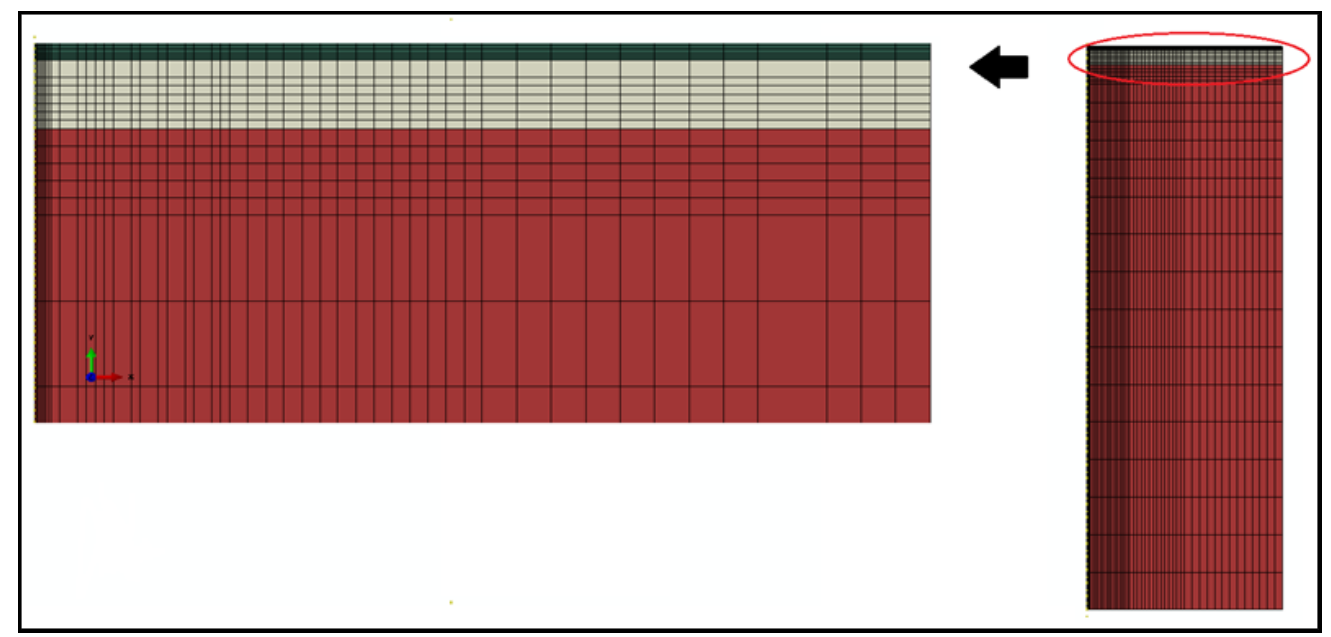

Figure 3. Mesh properties of FEM

The first step of the analysis is performed to verify the finite element model can generate the close to the same value as the elastic solution given the same assumptions against the elastic solution models. The results of surface deflections calculated by each model are presented in Figure 4 . Whilst an acceptable agreement is observed, the surface deflection determined by ABAQUS is less than what is determined by KENLAYER and CIRCLY. It can be interpreted that the finite element mesh is behaving slightly "stiffer" than the analytical solution. 


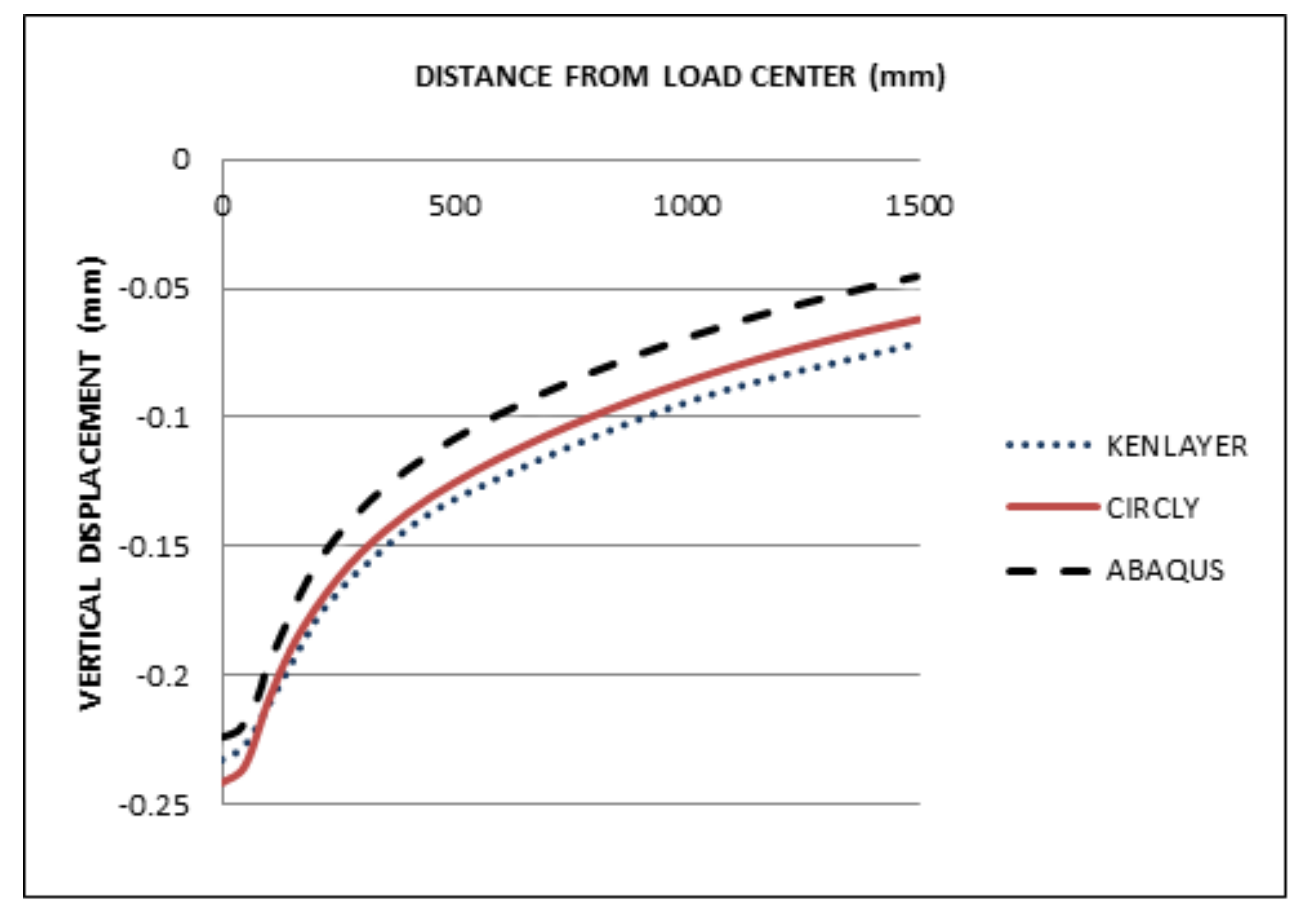

Figure 4. Surface deflection for the first model

\section{Nonlinear Model: Methodology and Characteristics}

Flexible pavement is one of the usual pavement structure used in WA. This makes it important to correctly analysis and model the behavior of this type of pavement. So far it has been a common trend to use linear elastic theory to model to analyze such a pavement, however, many research and studies has shown that the linear assumption is not fully representative of this type of pavement system.

There have been laboratory and field studies on the nonlinear behavior of granular materials used as base and subgrade in the flexible pavement ([22], [23], [24] and [25]).

The concept of resilient modulus has been developed to somehow account for this nonlinear behavior. Resilient modulus can be defined as the ratio between deviator stress and recoverable strain.

Here, nonlinear behavior means the change of materials elastic modulus according to the state of the stresses induced in the same material. One of the well-known equations to account for unbound granular materials nonlinearity is the Uzan [22] equation. In the axisymmetric condition (which is of interest of this paper) the Uzan [22] equation can be written as:

$$
M_{R}=K_{1}\left(\frac{I}{P_{0}}\right)^{k_{2}}\left(\frac{\sigma_{d}}{P_{0}}\right)^{k_{3}}
$$

where under axisymmetric assumption, the parameters are:

$\mathrm{I}=$ First invariant of stress tensor $=\sigma_{1}+2 \sigma_{2}$

$\sigma_{\mathrm{d}}=\sigma_{1}-\sigma_{2}$

$\mathrm{P}_{0}=$ Unit pressure (to make the equation dimensionally acceptable) $\mathrm{K}_{1}, \mathrm{~K}_{2}$ and $\mathrm{K}_{3}$ are materials parameters from laboratory test results

This equation can represent the hardening behavior of granular materials usually seen in the base layer. For the subgrade soil, which consists of fine grain soil, a bilinear model of Thompson and Robnett [26] is one the most acceptable models. This bilinear equation models the softening behavior of fine grain subgrade and can be written as follow: 


$$
\begin{array}{ll}
M_{R}=K_{1}+K_{\mathrm{a}} \times\left(K_{2}-\sigma_{d}\right): & \sigma_{d}<K_{2} \\
M_{R}=K_{1}-K_{4} \times\left(\sigma_{d}-K_{2}\right): & \sigma_{d}>K_{2}
\end{array}
$$

where:

$\mathrm{K}_{1}, \mathrm{~K}_{2}, \mathrm{~K}_{3}$ and $\mathrm{K}_{4}$ are materials parameters from laboratory test results

To implement a nonlinear analysis in this paper, a very simple and effective method has been applied. In this method the tyre load is equally divided and cumulatively applied in incremental steps. In each step, the stress state in base and subgrade layer is calculated separately. Having determined the stress of each layers in each step, then equation 3 and 4 are applied to calculate resilient modulus of layers in different depths. The average of this resilient modulus in each layer has been picked as the representative modulus of the layer during the next step. The procedure is continued until the full load of tyre has been applied and the results are calculated.

It is noted that this procedure is common among geotechnical engineers; however, there is no scholarly report using such a technique in the numerical analysis of pavements.

In the second part of this study, the second model is constructed in the ABAQUS program and for this new model both linear and nonlinear analyses are executed. Tables 2 and 3 illustrates the materials properties used for the second analysis. Here the same material characteristics and pavement layers used by Kim and Tutumluer [16] are chosen and results of the analysis are compared to their results.

\begin{tabular}{|c|c|c|c|}
\hline Layer & $\frac{\text { Thickness }}{(\mathrm{mm})}$ & $\frac{\text { Elastic Modulus }}{(\mathrm{MPa})}$ & $\frac{\text { Poisson }}{\underline{\text { Ratio }}}$ \\
\hline Asphalt (AC) & $\underline{76}$ & $\underline{2759}$ & $\underline{0.35}$ \\
\hline $\begin{array}{c}\text { Granular } \\
\text { (Base/Subbase) }\end{array}$ & $\underline{305}$ & 207/nonlinear & $\underline{0.4}$ \\
\hline Subgrade & 24619 & 41.4/nonlinear & $\underline{0.45}$ \\
\hline
\end{tabular}

Table 2. Material properties for the second model

A uniform loading pressure of $551 \mathrm{KPa}$ applied over a $152 \mathrm{~mm}$ circular area. This loading, is divided and then accumulatively applied over five subsequent steps. Except the materials characteristics and layer thickness, all the other properties in the second model are the same as the first model (Type of elements, boundary conditions and static loading).

Table 3. Material parameters used for nonlinear moduli

\begin{tabular}{ccccc}
\hline$\underline{\text { Layer }}$ & $\underline{\mathrm{K}_{1}}$ & $\underline{\mathrm{K}_{2}}$ & $\underline{\mathrm{K}_{3}}$ & $\underline{\mathrm{K}_{4}}$ \\
\hline$\underline{\text { Base (Eq. 3) }}$ & $\underline{4.1(\mathrm{MPa})}$ & $\underline{0.64}$ & $\underline{0.065}$ & - \\
$\underline{\text { Subgrade (Eq. 4) }}$ & $\underline{41.4(\mathrm{MPa})}$ & $\underline{0.041(\mathrm{MPa})}$ & $\underline{1000}$ & $\underline{200}$ \\
\hline
\end{tabular}

Figure 5 illustrates the surface deflection in two cases of Linear and nonlinear analysis. 


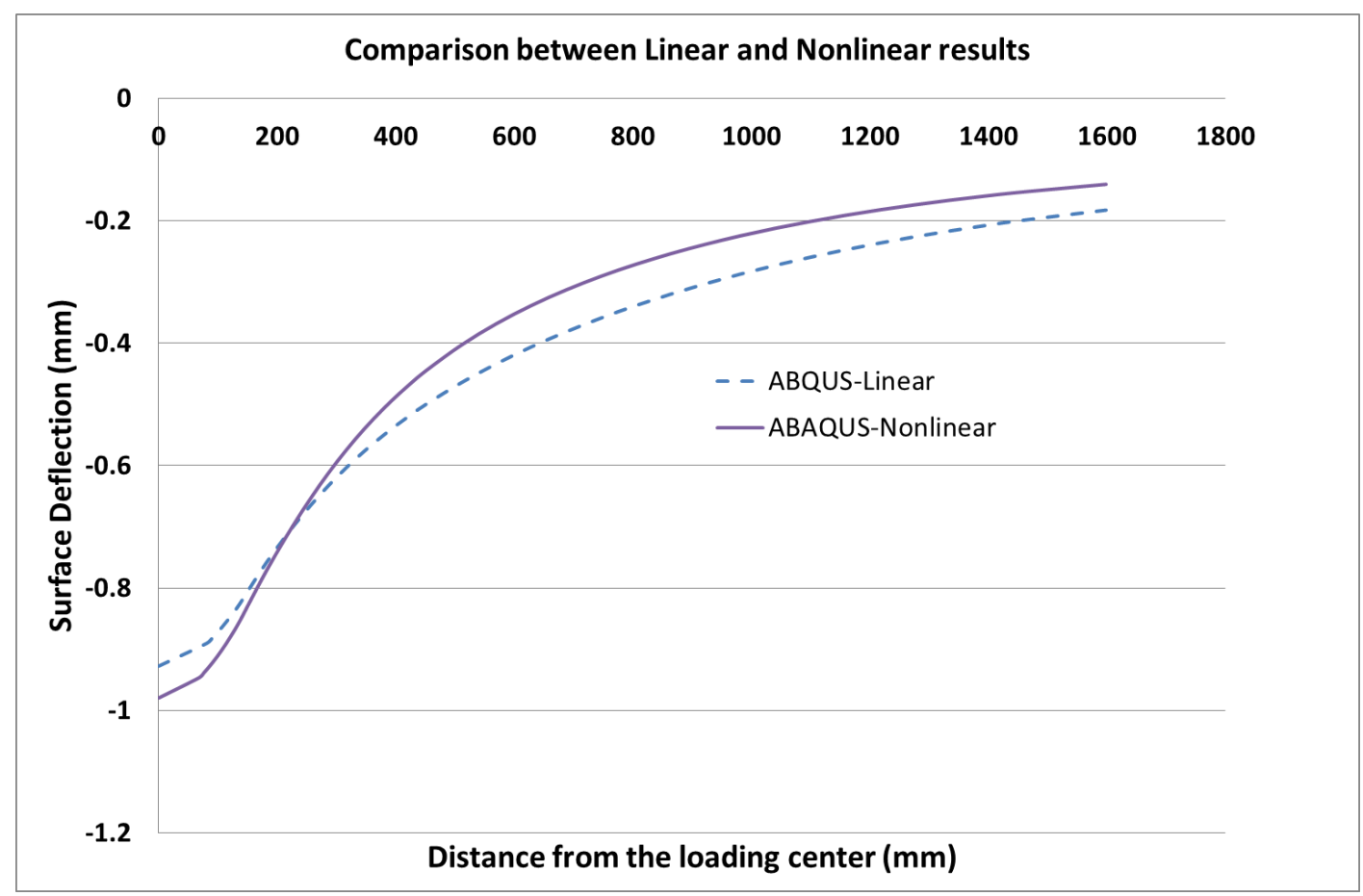

Figure 5. Surface deflection for the second model

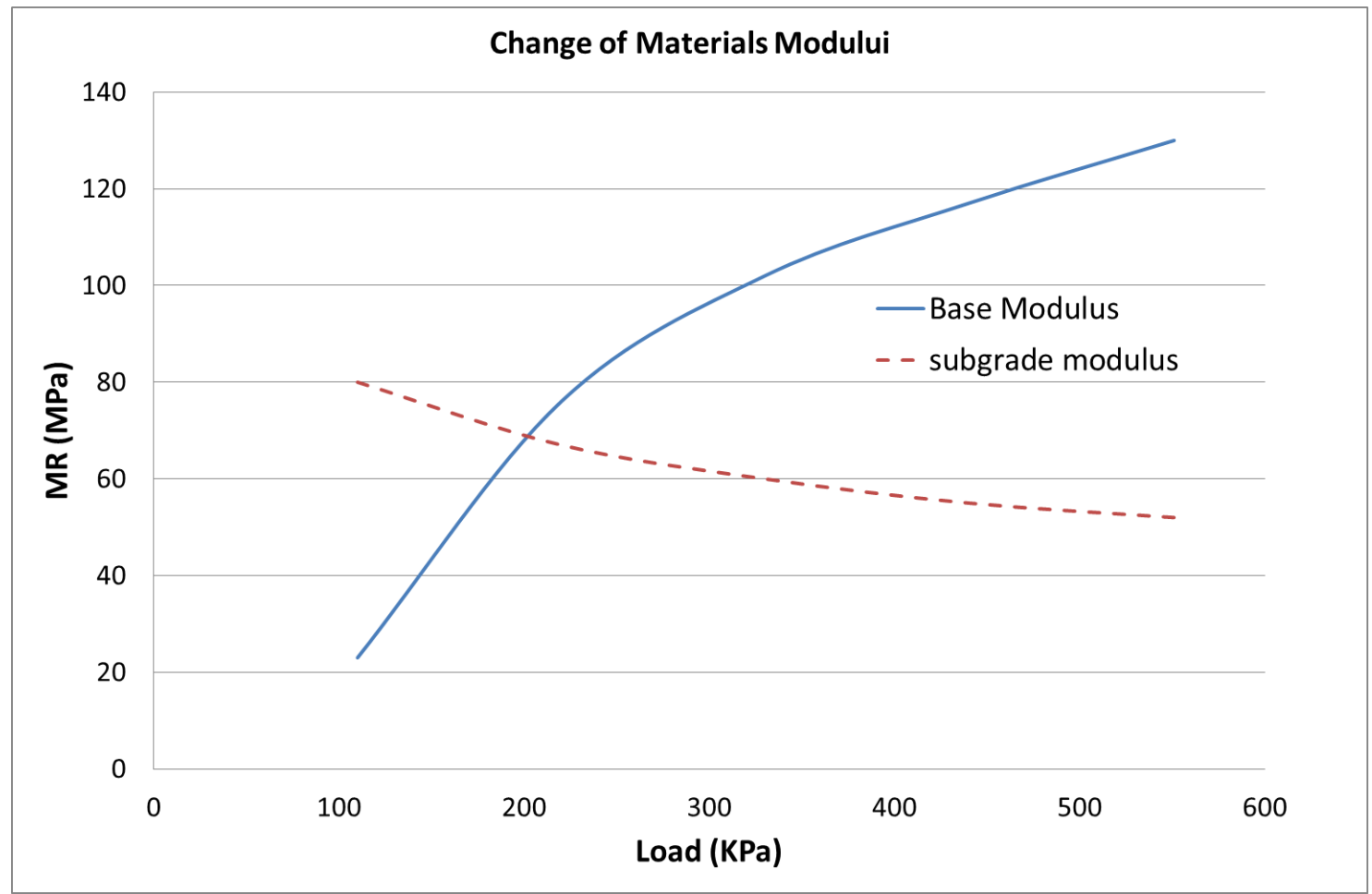

Figure 6. Gradual change in the resilient modulus of the layers

As the stress state is gradually increased, the nonlinear resilient moduli for the base and subgrade layers are produced. The base is modeled to show hardening behavior while subgrade has a softening trend which is characteristics of fine grain soils. This can be seen in Figure 6. Here resilient modulus of the base and subgrade layers are calculated according to equations 3 and 4 respectively.

Finally, Table 4 demonstrates the comparison of critical pavement responses between linear and nonlinear analysis. These are surface deflection of the asphalt layer beneath the loading, horizontal strain at the bottom of asphalt layer and vertical strain at the top of the subgrade. The results of [16] for the nonlinear analysis of the same model are represented as well. 
It is apparent that nonlinear analysis results in larger deformation and strains. This especially has an effect in the mechanistic design of the pavement layers. According to this analysis an increase of $6 \%$ in the surface deflection is calculated. Tensile strain at the bottom of asphalt layer shows a more severe increase (33\%). Finally the vertical strain at the top of subgrade has increased by $6 \%$.

[16] have reported a $4 \%$ increase for the surface deflection, $13 \%$ increase for the horizontal strain at the bottom of asphalt layer and $0.4 \%$ increase for the vertical strain at the top of subgrade.

Table 4. Material properties for the second model

\begin{tabular}{|c|c|c|c|}
\hline Critical Response & Linear & Nonlinear & $\frac{\text { Kim and }}{\underline{\text { Tutumluer }}} \frac{(2009)}{\underline{(20)}}$ \\
\hline$\frac{\delta(\mathrm{mm})}{\underline{\text { surface }}}$ & $\underline{-0.928}$ & $\underline{-0.980}$ & $\underline{-0.968}$ \\
\hline$\underline{\varepsilon}_{\mathrm{h}} \frac{\text { (microstrains) }}{\text { bottom of } \mathrm{AC}}$ & $\underline{361}$ & $\underline{480}$ & $\underline{257}$ \\
\hline$\underline{\varepsilon}_{\mathrm{V}} \frac{\text { (microstrains) }}{\text { top of } \mathrm{SG}}$ & $\underline{967}$ & $\underline{1023}$ & 937 \\
\hline
\end{tabular}

\section{Summary}

Two systems of layered flexible pavements have been constructed. The first model is analyzed through KENLAYER, CIRCLY and ABAQUS. While the first two of these programs use the elastic theory the ABAQUS program uses the finite element technique. Then the second model is constructed in ABAQUS and analyzed through linear elastic and nonlinear elastic assumptions. A simple methodology has been applied through which loading is divided in 5 subsequent steps and the resilient modulus of each step has been calculated according to stress state of the layer. Results show a larger deformation, larger tensile strain at the bottom of asphalt layer and larger vertical strain at the top of subgrade. The maximum of $33 \%$ difference has been calculated for the horizontal strain at the asphalt layer.

\section{References}

[1]. Huang, Y.H., Pavement analysis and design1993: Prentice-Hall Inc., New Jersey, USA.

[2]. Huang, Y.H., Pavement analysis and design2004: Pearson Prentice Hall, Pearson Education Inc., USA.

[3]. Gedafa, D.S., Comparison of Flexible Pavement Performance Using Kenlayer and HDM-4. Midwest Transportation Consortium, Ames, Iowa, 2006.

[4]. Wardle, L., Program CIRCLY: A Computer Program for the Analysis of Multiple Complex Circular Loads on Layered Anisotropic Media. User's Manual1977: Division of Applied Geomechanics, Commonwealth Scientific and Industrial Research Organization Australia.

[5]. Bodhinayake, B.C., A study on nonlinear behaviour of subgrades under cyclic loading for the development of a design chart for flexible pavements. University of Wollongong Thesis Collection, 2008: p. 868.

[6]. Ullidtz, P. Analytical tools for design of flexible pavements. in Keynote Address. In: Proceedings if the 9th International Conference on Asphalt Pavements, . 2002. Copenhagen, . 
[7]. Wardle, L., G. Youdale, and B. Rodway. Current issues for mechanistic pavement design. in 21st ARRB and 11th REAAA Conference, . 2003. Cairns, Australia, : ARRB Transport Research.

[8]. Hadi, M. and M. Symons, Computing stresses in road pavements using CIRCLY, MSC/NASTRAN and STRAND6. Transactions of the Institution of Engineers, Australia. Civil engineering, 1996. 38(2-4): p. 89-93.

[9]. Tutumluer, E., D.N. Little, and S.H. Kim, Validated model for predicting field performance of aggregate base courses. Transportation Research Record: Journal of the Transportation Research Board, 2003. 1837(-1): p. 41-49.

[10]. Zaghloul, S. and T. White, Use of a three-dimensional, dynamic finite element program for analysis of flexible pavement. Transportation Research Record, 1993(1388): p. 60-69.

[11]. Mallela, J. and K. George, Three-dimensional dynamic response model for rigid pavements. Transportation Research Record, 1994(1448).

[12]. Uddin, W., D. Zhang, and F. Fernandez, Finite element simulation of pavement discontinuities and dynamic load response. Transportation Research Record, 1994(1448).

[13]. Cho, Y.H., B.F. McCullough, and J. Weissmann, Considerations on finite-element method application in pavement structural analysis. Transportation Research Record: Journal of the Transportation Research Board, 1996. 1539(-1): p. 96-101.

[14]. Kim, M. and E. Tutumluer. Modeling Nonlinear, Stress-Dependent Pavement Foundation Behavior Using a General-Purpose Finite Element Program. 2006. ASCE.

[15]. Kim, M., Three-dimensional finite element analysis of flexible pavements considering nonlinear pavement foundation behavior, 2007, University of Illinois.

[16]. Kim, M., E. Tutumluer, and J. Kwon, Nonlinear pavement foundation modeling for three-dimensional finite-element analysis of flexible pavements. International Journal of Geomechanics, 2009. 9: p. 195.

[17]. Vuong, B.T., Validation of a three-dimensional nonlinear finite element model for predicting response and performance of granular pavements with thin bituminous surfaces under heavy vehicles. Road \& Transport Research: A Journal of Australian and New Zealand Research and Practice, 2008. 17(1): p. 3.

[18]. Duncan, J.M., C.L. Monismith, and E.L. Wilson, Finite element analyses of pavements. Highway Research Record Highway Research Board, Washington D. C., 1968. 228: p. 18-33.

[19]. Huang, Y. Finite element analysis of nonlinear soil media in Proceedings, Symposium on Application of Finite Element Methods in Civil Engineering, . 1969. Vanderbilt University, Nashville, The Netherlands.

[20]. AUSTROADS, Pavement Design - A Guide to the Structural Design of Road Pavements2004: Austroads, Sydney, Australia.

[21]. Yu, H.S., Plasticity and geotechnics. Vol. 13. 2006: Springer. 
[22]. Uzan, J., Characterization of granular material. Transportation Research Record, 1985(1022).

[23]. Tutumluer, E., Predicting behavior of flexible pavements with granular bases, 1995.

[24]. Rowshanzamir, M.A., Resilient Cross-anisotropic Behaviour of Granular Base Materials Under Repetitive Loading, 1997, University of New South Wales: Sidney

[25]. Jooste, F.J. and E. Fernando, Development of a procedure for the structural evaluation of superheavy load routes, 1995.

[26]. Thompson, M.R. and Q.L. Robnett, Resilient properties of subgrade soils. Transportation Engineering Journal, 1979. 105(1): p. 71-89. 